\title{
Reoperation after failed resective epilepsy surgery in children
}

\author{
*Osama Muthaffar, MD, ${ }^{1,2}$ Klajdi Puka, HBSc, ${ }^{3}$ Luc Rubinger, HBMSc, ${ }^{4}$ Cristina Go, MD, ${ }^{1}$ \\ O. Carter Snead III, MD, ${ }^{1}$ James T. Rutka, MD, PhD, ${ }^{5}$ and Elysa Widjaja, MD ${ }^{1,6}$
}

\begin{abstract}
${ }^{1}$ Division of Neurology; Departments of ${ }^{3}$ Psychology, ${ }^{5}$ Neurosurgery, and ${ }^{6}$ Diagnostic Imaging; ${ }^{4}$ Neuroscience and Mental Health, Hospital for Sick Children, Toronto, Ontario, Canada; and ${ }^{2 D i v i s i o n}$ of Pediatrics, King Abdulaziz University Hospital, Jeddah, Saudi Arabia
\end{abstract}

\begin{abstract}
OBJECTIVE Although epilepsy surgery is an effective treatment option, at least $20 \%-40 \%$ of patients can continue to experience uncontrolled seizures resulting from incomplete resection of the lesion, epileptogenic zone, or secondary epileptogenesis. Reoperation could eliminate or improve seizures. Authors of this study evaluated outcomes following reoperation in a pediatric population.
\end{abstract}

METHODS A retrospective single-center analysis of all patients who had undergone resective epilepsy surgery in the period from 2001 to 2013 was performed. After excluding children who had repeat hemispherotomy, there were 24 children who had undergone a second surgery and 2 children who had undergone a third surgery. All patients underwent $\mathrm{MRI}$ and video electroencephalography (VEEG) and 21 underwent magnetoencephalography (MEG) prior to reoperation.

RESULTS The mean age at the first and second surgery was 7.66 (SD 4.11) and 10.67 (SD 4.02) years, respectively. The time between operations ranged from 0.03 to 9 years. At reoperation, 8 patients underwent extended cortical resection; 8, lobectomy; 5, lesionectomy; and 3, functional hemispherotomy. One year after reoperation, $58 \%$ of the children were completely seizure free (International League Against Epilepsy [ILAE] Class 1) and 75\% had a reduction in seizures (ILAE Classes 1-4). Patients with MEG clustered dipoles were more likely to be seizure free than to have persistent seizures $(71 \%$ vs $40 \%, p=0.08)$.

CONCLUSIONS Reoperation in children with recurrent seizures after the first epilepsy surgery could result in favorable seizure outcomes. Those with residual lesion after the first surgery should undergo complete resection of the lesion to improve seizure outcome. In addition to MRI and VEEG, MEG should be considered as part of the reevaluation prior to reoperation.

https://thejns.org/doi/abs/10.3171/2017.3.PEDS16722

KEY WORDS pediatric epilepsy surgery; reoperation; repeat surgery; magnetoencephalography; outcomes

$\mathrm{F}$ OR patients who have been unable to attain adequate seizure control from at least 2 trials of antiepileptic drugs (AEDs), epilepsy surgery is a very effective treatment option leading to seizure control in approximately $60 \%-80 \%$ of cases $^{28}$ However, the remaining $20 \%-$ $40 \%$ of cases and $54 \%-73 \%$ of patients with extratemporal lobe resections continue to have uncontrolled seizures. ${ }^{30}$ These persistent seizures may originate from residual epileptogenic tissue, as a result of limited resection, or from secondary epileptogenesis. ${ }^{5,10,25-27,34}$ Therefore, some patients may benefit from a second surgery excising the remaining epileptogenic tissue. Indeed, of the $6 \%-23 \%$ of patients who undergo reoperation, $9.5 \%-87 \%$ attain sei- zure freedom. ${ }^{2,5,9,17,21,23,26,29,33}$ However, this rate of seizure freedom should be considered with caution as the patients who undergo reoperation were judged to be eligible for reoperation and had a good chance of attaining seizure control. Additionally, patient characteristics and surgery type greatly influence outcomes. Prior studies have shown that predictors of favorable seizure control following reoperation include the removal of recurrent tumor, ${ }^{25}$ persistent focal interictal discharges on scalp electroencephalography (EEG) ${ }^{27}$ and extension of the previous resection based on ictal EEG recordings that were concordant with ictal EEG and MRI findings before the first epilepsy surgery ${ }^{10}$ as well as resection of magnetoencephalography (MEG) clusters

ABBREVIATIONS AED = antiepileptic drug; EEG = electroencephalography; $F C D=$ focal cortical dysplasia; ILAE = International League Against Epilepsy; $M E G=$ magnetoencephalography; VEEG = video-EEG; VNS = vagus nerve stimulator.

SUBMITTED December 28, 2016. ACCEPTED March 13, 2017.

INCLUDE WHEN CITING Published online June 2, 2017; DOI: 10.3171/2017.3.PEDS16722.

* Dr. Muthaffar and Mr. Puka contributed equally to this work. 
adjacent to the margins of the previous resection in addition to removing recurrent lesions. ${ }^{16}$

There are few studies on reoperation for pediatric epilepsy surgery. ${ }^{2,21,26}$ The aims of this study were to assess outcomes following reoperation for pediatric epilepsy at a tertiary care pediatric epilepsy center and to evaluate the factors associated with favorable seizure control. Identifying such factors is important in selecting appropriate patients who may benefit from reoperation and in providing diagnostic indicators of possible outcomes.

\section{Methods \\ Patients}

We performed a retrospective health record analysis of all patients who had undergone resective epilepsy surgery at the Hospital for Sick Children in Toronto, Ontario, Canada, between January 1, 2001, and December 31, 2013. All patients were 18 years of age or younger at the time of surgery. Exclusion criteria included nonresective surgeries (such as placement of vagus nerve stimulators [VNSs], corpus callosotomy, or invasive monitoring without resection) and patients lost to follow-up in the following year. A total of 309 children were identified and had resective surgery, as detailed previously. ${ }^{22}$ Of these children, 198 (64\%) were seizure free 1 year later, and $111(36 \%)$ had persistent seizures. There were 33 children (11\% of the entire sample) who underwent repeat epilepsy surgery. Eight patients underwent repeat hemispherotomy, and the outcomes of these patients have been reported elsewhere ${ }^{14}$ hence, these patients were excluded from this study. One patient was lost to follow-up. The final cohort consisted of 24 children who underwent repeat surgery and were followed up for at least 1 year after reoperation.

\section{Clinical Data Collection}

The demographics, age at seizure onset, seizure semiology, seizure frequency before and after surgery, and number of AEDs were extracted from patients' medical charts. Surgery-related data were collected for the first surgery and reoperation and included the date of epilepsy surgery, type and location of surgery, and histological findings. This study was approved by the Research Ethics Board of the Hospital for Sick Children, and the study data were managed and stored using REDCap. ${ }^{8}$

\section{Magnetic Resonance Imaging, MEG, and Video-EEG}

All patients underwent 1.5-T (patients evaluated before 2008) or 3.0-T MRI (patients evaluated after 2008) prior to the first surgery and reoperations, as detailed previously. ${ }^{22}$ These studies included axial and coronal T2-weighted, axial and coronal FLAIR, axial and coronal proton density (PD), and volumetric T1-weighted images. Diffusion tensor imaging was also performed in patients who had had hemispherectomy.

All patients underwent scalp video-EEG (VEEG; Harmonie 5.4 and NeuroWorks 8.0, Natus Medical Inc.), which was recorded using 19 or 25 scalp electrodes placed according to the international 10-10 system (subtemporal electrodes F9, F10, T9, T10, P9, and P10; or midline electrodes F1, F2, C1, C2, P1, and P2). A single reference was placed at $\mathrm{Oz}, \mathrm{Pz}^{\prime}$ (located $1 \mathrm{~cm}$ behind $\mathrm{Pz}$ ), or FCz, depend- ing on which was the most inactive electrode. Sampling rate was 200,500 , or $1000 \mathrm{~Hz}$. Prolonged scalp VEEG studies were performed in conjunction with electromyography recordings from both deltoid muscles without recording from chin and electrooculography electrodes. All patients had VEEG studies before the first epilepsy surgery and reoperations.

Magnetoencephalography was done using a whole-head Omega 151-channel gradiometer system (VSM MedTech). The MEG recording procedures and methods for detecting, localizing, and analyzing interictal MEG spikes have been described previously. ${ }^{1-13}$ The MEG spike source cluster was defined as a localized group of 6 or more spike sources with $1 \mathrm{~cm}$ or less between adjacent sources; MEG scatter was defined as a group of less than 6 spike sources regardless of the distance between the spike sources, or as a group of spike sources with more than $1 \mathrm{~cm}$ between each spike source regardless of the number of spike sources. ${ }^{13}$ Magnetoencephalography clustered dipoles have been shown to correspond to the epileptogenic zone. ${ }^{12}$

\section{Epilepsy Surgery Outcome}

Seizure outcome was classified using the International League Against Epilepsy (ILAE) classification: ${ }^{32}$ Class 1, completely seizure free with no auras; Class 2 , auras only with no seizures; Class 3, 1-3 seizure days per year with or without auras; Class 4, 4 seizure days per year to $50 \%$ reduction of baseline seizure days with or without auras; Class 5, less than $50 \%$ reduction of baseline seizure days to $100 \%$ increase of baseline seizure days with or without auras; and Class 6, more than $100 \%$ increase of baseline seizure days with or without auras. Seizure outcome after the first surgery and reoperation was categorized as seizure free (ILAE Class 1) versus persistent seizures (ILAE Class 2-6). We also compared patients who showed postoperative seizure improvement (ILAE Class 1-4) with patients who showed no improvement (ILAE Class 5-6).

\section{Statistical Analyses}

Analyses were conducted using IBM SPSS Statistics for Windows version 23.0 (IBM Corp.). Descriptive statistics used to describe the sample included mean and standard deviation for continuous measures and frequency and percentage for categorical variables. Independent samples t-tests, chi-square analyses, or Fisher's exact test was used to compare patient and epilepsy-related variables between those who attained seizure control following reoperation and those who experienced persistent seizures.

\section{Results}

\section{Patient Characteristics and Examinations Prior to the First Surgery}

Table 1 features the demographic and epilepsy-related variables of the 24 children included in this study. The mean age at seizure onset was $4.2 \pm 3.7$ years, and the mean age at the first surgery was $7.7 \pm 4.1$ years. At baseline, children were taking between 1 and 4 AEDs (mean 2.2 $\pm 0.9), 17$ children $(71 \%)$ had daily seizures, $5(21 \%)$ had weekly seizures, 1 (4\%) had monthly seizures, and 1 (4\%) had yearly seizures. All children had undergone a second 
TABLE 1. Summary of patient demographics and examinations before and types of first and second surgeries

\begin{tabular}{|c|c|}
\hline Parameter & Value \\
\hline Total no. of patients & 24 \\
\hline Female sex & $10(41.7 \%)$ \\
\hline Age at seizure onset in yrs & $4.20 \pm 3.74$ \\
\hline Age at 1 st surgery in yrs & $7.66 \pm 4.11$ \\
\hline Age at $2 n d$ surgery in yrs & $10.67 \pm 4.02$ \\
\hline No. of preop AEDs & $2.21 \pm 0.88$ \\
\hline \multicolumn{2}{|l|}{ Site of seizure focus } \\
\hline Temporal & $12(50 \%)$ \\
\hline Extratemporal & $10(42 \%)$ \\
\hline Temporal \& extratemporal & $2(8 \%)$ \\
\hline Normal preop MRI & $2(8 \%)$ \\
\hline \multicolumn{2}{|l|}{ Histology } \\
\hline FCD & $8(33 \%)$ \\
\hline Tumor & $7(29 \%)$ \\
\hline Rasmussen encephalitis & $2(8 \%)$ \\
\hline Other & $5(21 \%)$ \\
\hline No discernable pathology & $2(8 \%)$ \\
\hline \multicolumn{2}{|l|}{ EEG findings before 1st surgery } \\
\hline Unilat focal & $11(46 \%)$ \\
\hline Unilat multifocal & $11(46 \%)$ \\
\hline Unilat hemispheric & $1(4 \%)$ \\
\hline Bilat & $1(4 \%)$ \\
\hline \multicolumn{2}{|l|}{ Seizure semiology prior to 1st surgery } \\
\hline Complex partial w/o $2^{\circ}$ generalization & $17(71 \%)$ \\
\hline Complex partial w/ $2^{\circ}$ generalization & $1(4 \%)$ \\
\hline Simple partial w/o $2^{\circ}$ generalization & $3(12 \%)$ \\
\hline Others & $3(12 \%)$ \\
\hline Similar seizure semiology after 1 st surgery & $15(62 \%)$ \\
\hline Clustered dipoles on MEG before 1st surgery* & $17(77 \%)$ \\
\hline Clustered dipoles on MEG before 2 nd surgery $\dagger$ & $14(67 \%)$ \\
\hline Invasive monitoring before 1st surgery & $7(29 \%)$ \\
\hline Invasive monitoring before 2nd surgery & $7(29 \%)$ \\
\hline Residual lesion identified after 1st surgery & $7(29 \%)$ \\
\hline Residual lesion identified after 2nd surgery & $2(8 \%)$ \\
\hline \multicolumn{2}{|l|}{ Type of 1st surgery } \\
\hline Lesionectomy & $11(46 \%)$ \\
\hline Cortical resection (w/ or w/o lesion resection) & $10(42 \%)$ \\
\hline Lobectomy & $3(12 \%)$ \\
\hline \multicolumn{2}{|l|}{ Type of 2nd surgery } \\
\hline Lesionectomy (\& recurrent tumor) & $5(21 \%)$ \\
\hline Extended cortical resection & $8(33 \%)$ \\
\hline Lobectomy & $8(33 \%)$ \\
\hline Functional hemispherotomy & $3(12.5 \%)$ \\
\hline $\begin{array}{l}2^{\circ}=\text { secondary. } \\
\text { Values expressed as mean } \pm \text { SD or as number }(\%) \text {. } \\
{ }^{*} \text { Magnetoencephalography was completed for } 22 \text { childre } \\
\dagger \text { Magnetoencephalography was completed for } 21 \text { childr }\end{array}$ & \\
\hline
\end{tabular}

resective surgery at a mean age of $10.7 \pm 4.0$ years, with an interval of 13 days to 9 years after the initial surgery (mean $3.0 \pm 2.4$ years). Two children had undergone a third resective surgery at the age of $15.3 \pm 0.3$ years $(2.18-2.38$ years after the second surgery).

Prior to the first surgery, most children (17 [71\%]) had complex partial seizures without secondary generalization; few children had simple partial seizures without secondary generalization (3 [12\%]), complex partial seizures with secondary generalization (1 [4\%]), generalized seizures (2 [8\%]), or other seizure types (1 [4\%]).

Among the 24 children, $22(92 \%)$ had abnormal MRI findings. Seventeen (77\%) of 22 children had clustered dipoles on MEG. Eleven (46\%), $11(46 \%), 1(4 \%)$, and $1(4 \%)$ had unilateral focal, multilobar, bilateral, or hemispheric EEG ictal/interictal discharges, respectively. The most common pathological lesions were focal cortical dysplasia $(\mathrm{FCD} ; 8[33 \%])$ and tumor $(7[29 \%])$. A roughly equal number of lesionectomies (11 [46\%]) and cortical resections $(10[42 \%])$ and fewer lobectomies $(3[12 \%])$ were completed at the first surgery.

\section{Postoperative Examinations and Type of Surgery}

After the first surgery, seizure semiology was similar in 15 children (62\%). Twenty-one children (88\%) had undergone MEG prior to the second surgery; of these, 14 $(67 \%)$ had clustered MEG dipoles ipsilateral to the previous resection, 6 (28\%) had scattered dipoles, and 1 (5\%) did not show dipole on MEG. Seven patients underwent lesionectomy for the first surgery and did not subsequently have residual lesion. These 7 patients underwent MEG and 6 showed MEG clustered dipoles, but none had invasive monitoring.

At reoperation, 8 children (33\%) underwent lobectomy; of these, $4(50 \%)$ had undergone a previous cortical resection and $4(50 \%)$ had undergone a previous lesionectomy (Table 2). Eight children (33\%) underwent extended cortical resection at reoperation; of these, $3(37.5 \%)$ had undergone a previous cortical resection, $3(37.5 \%)$ had a previous lesionectomy, $1(12.5 \%)$ had a previous resection of an MEG dipole cluster adjacent to a porencephalic cyst, and 1 $(12.5 \%)$ had a previous lobectomy. Three children (12.5\%) underwent a functional hemispherotomy at reoperation; 2 $(67 \%)$ had undergone a previous lobectomy and 1 (33\%) had a previous cortical resection. Seven patients (29\%) had invasive monitoring prior to the first surgery, and none of them had invasive monitoring prior to reoperation. Of the 17 patients $(71 \%)$ who did not have invasive monitoring prior to the first surgery, $7(41 \%)$ had invasive monitoring prior to reoperation; 5 (71\%) of these patients had extended cortical resection and $2(29 \%)$ had lobectomy at reoperation.

Magnetic resonance imaging prior to the second surgery showed that 7 patients (29\%) had residual lesions: 3 (43\%) were residual tumor, 3 (43\%) were residual FCD, and $1(14 \%)$ was hypothalamic hamartoma. Of these 7 patients, 5 underwent repeat surgery within 2 years, and 2 underwent repeat surgery more than 5 years later. One patient who underwent repeat surgery 6.4 years later had residual FCD immediately adjacent to the leg motor area and had a vagus nerve stimulator (VNS) inserted after an unsuc- 
TABLE 2. Types of first and second surgeries

\begin{tabular}{lcccc}
\hline & & \multicolumn{3}{c}{ Second Surgery } \\
\cline { 2 - 5 } 1st Surgery & Extended Cortical Resection & Functional Hemispherotomy & Lesionectomy & Lobectomy \\
\hline Resection of MEG cluster & $1^{*}$ & 0 & 0 & 0 \\
\hline Cortical resection & 3 & 1 & $1 \dagger$ & 4 \\
\hline Lesionectomy & 3 & 0 & $4 \ddagger$ & 0 \\
\hline Lobectomy & 1 & 2 & 4 \\
\hline
\end{tabular}

* One patient underwent resection of an MEG cluster adjacent to a porencephalic cyst at the first surgery, which failed. This patient underwent invasive monitoring and resection of the epileptogenic cortex, guided by subdural grid data at the second surgery.

$\dagger$ One patient underwent invasive monitoring and had resection of FCD immediately adjacent to the leg motor area and epileptogenic cortex as defined by invasive monitoring at the first surgery. There was residual FCD, which was resected at the second surgery.

$\ddagger$ Three patients had resection of residual tumor or hypothalamic hamartoma, and 1 patient had resection of recurrent tumor at the second surgery.

cessful first surgery. The patient continued to have seizures despite the VNS, had repeat surgery to remove the residual FCD, and was seizure free following the repeat surgery. The second patient who had repeat surgery 9 years later had a large dysembryoplastic neuroectodermal tumor (DNET) that involved the basal ganglia, thalamus, and cerebral peduncle, and repeat surgery was done as a palliative procedure. Four patients $(17 \%)$ who had incomplete resection of tumor or hypothalamic hamartoma underwent further lesionectomy, and 1 patient (4\%) underwent resection of recurrent tumor at reoperation. Following the second surgery, 2 patients (8\%) had residual lesion: one had an extensive DNET that involved the basal ganglia, thalamus, and cerebral peduncle, and the other had residual hypothalamic hamartoma. Of the 3 patients $(12.5 \%)$ with residual FCD, 2 underwent lesionectomy and 1 underwent extended cortical resection including resection of remnant FCD. Four patients (57\%) with residual lesion after the first surgery were seizure free and $1(14 \%)$ experienced improvement in seizures. Two patients did not experience seizure reduction following the second surgery: one had residual hypothalamic hamartoma after the second surgery, and the other had persistent seizures despite complete removal of residual FCD.

\section{Reoperation: Epilepsy Surgery Outcome}

Table 3 provides a summary of the seizure outcomes 1 and 2 years following the second and third surgery. One year after the second surgery, 14 children $(58 \%)$ were completely seizure free (ILAE Class 1), and 18 children (75\%) had improved seizures (ILAE Class 1-4). There were 18 children (75\%) who were followed up 2 years after the reoperation; of these, $10(56 \%)$ were seizure free (ILAE Class 1) and 15 (83\%) had improved seizures (ILAE Class $1-4)$ since the second surgery. Patients who had not attained seizure control 1 year after the reoperation continued to have seizures in future follow-up visits. The number of AEDs following reoperation was similar at the 1- and 2-year follow-ups; the median number of medications at each time point was 2.

There were 2 patients $(8 \%)$ who underwent a third surgery. One child with FCD underwent invasive monitoring with cortical resection and lesionectomy at the first surgery, followed by extended cortical resection and resection of residual FCD at the second surgery, and subsequent functional hemispherotomy at the third surgery, which rendered the patient seizure free. The second child developed gliosis following choroid papilloma removal, which resulted in epilepsy, and underwent limited cortical resection for the first epilepsy surgery, followed by temporal lobectomy at the second surgery and extended cortical resection beyond the lobectomy site at the third surgery. This patient continued to have seizures following the third surgery. Both patients were on 3 AEDs at the 1- and 2-year follow-ups.

Table 4 presents a comparison of the patient and epilepsy-related variables between children who were seizure free 1 year following reoperation and children who had persistent seizures. There was a trend for patients with MEG clustered dipoles prior to the second surgery to be seizure free $(10[71 \%]$ of 14$)$ rather than to have persistent seizures $(4[40 \%]$ of 10$)$ after reoperation $(p=0.080$, Fisher's exact test). Age at seizure onset, age at first or second surgery, site of seizure focus, histology, having similar seizure semiology after the first surgery, invasive monitoring at the second surgery, and type of second surgery were not associated with a seizure-free outcome.

\section{Discussion}

We reviewed results of examinations and surgical outcomes of children who had undergone reoperation for epilepsy surgery at a large tertiary care center in Canada. Of the 309 patients who had undergone resective epilepsy surgery at our center, 24 (8\%) had a second operation, after excluding repeat hemispherotomy cases. This rate is similar to the reoperation rate found across other centers, which ranges from $6 \%$ to $23 \% \%^{2,5,21,26,29,33}$ In the current study, we found that $58 \%$ of children were seizure free (ILAE Class 1) and 75\% had a significant reduction of seizures (ILAE Class 1-4) 1 year after reoperation. The number of AEDs remained similar, in accordance with the practice at our institution of maintaining AEDs for at least 2 years following seizure control. These results are similar to those in previous studies finding that $44 \%-87 \%$ of children attain seizure freedom following reoperation. ${ }^{2,5}$, 21,26,29,33 Importantly, we found that patients who had not attained seizure control 1 year after reoperation continued to have seizures in future follow-up visits.

There could be several reasons for the failed first surgery. Seven patients had residual lesions (tumor, FCD, or hypothalamic hamartoma), and 5 of these patients became 
TABLE 3. Seizure outcomes and medications following reoperations

\begin{tabular}{|c|c|c|}
\hline Parameter & Value & Range \\
\hline \multicolumn{3}{|l|}{ Second Surgery } \\
\hline Age at 2nd surgery in yrs & $10.67 \pm 4.02$ & $2.16-18.03$ \\
\hline Time from 1 st to 2 nd surgery in yrs & $3.00 \pm 2.43$ & $0.03-9.00$ \\
\hline \multicolumn{3}{|l|}{1 yr after $2 n d$ surgery $(n=24)$} \\
\hline Seizure free (ILAE Class 1) & $14(58 \%)$ & \\
\hline $\begin{array}{l}\text { Seizures improved (ILAE Class } \\
1-4)\end{array}$ & $18(75 \%)$ & \\
\hline No. of AEDs & $2.38 \pm 0.92$ & $1-4$ \\
\hline \multicolumn{3}{|l|}{2 yrs after 2 nd surgery $(n=18)$} \\
\hline Seizure free (ILAE Class 1) & $10(56 \%)$ & \\
\hline $\begin{array}{l}\text { Seizures improved (ILAE Class } \\
1-4)\end{array}$ & $15(83 \%)$ & \\
\hline No. of AEDs & $1.94 \pm 1.21$ & $0-5$ \\
\hline \multicolumn{3}{|l|}{ Third Surgery } \\
\hline Age at 3rd surgery in yrs & $15.31 \pm 0.30$ & $15.09-15.52$ \\
\hline Time from 2nd to 3 rd surgery in yrs & $2.28 \pm 0.14$ & $2.18-2.38$ \\
\hline \multicolumn{3}{|l|}{1 yr after 3rd surgery $(n=2)$} \\
\hline Seizure free (ILAE Class 1) & $1(50 \%)$ & \\
\hline $\begin{array}{l}\text { Seizures improved (ILAE Class } \\
1-4)\end{array}$ & $2(100 \%)$ & \\
\hline No. of AEDs & 3 & - \\
\hline \multicolumn{3}{|l|}{2 yrs after 3rd surgery $(n=1)$} \\
\hline Seizure free (ILAE Class 1) & $1(100 \%)$ & \\
\hline No. of AEDs & 3 & - \\
\hline
\end{tabular}

$\mathrm{n}=$ number of patients.

Values expressed as mean \pm SD or as number (\%).

seizure free or had improvement in seizures following reoperation for resection of the residual lesion. The epileptogenic zone may have been more extensive than the MRI visible lesion. Hence, lesionectomy alone is insufficient to render patients free of seizures. In these patients, MEG prior to the reoperation may help to clarify the extent of the epileptogenic zone. In 7 of our patients who had lesionectomy at the first surgery but did not have residual lesion, MEG was performed, which guided the second surgery. Other reasons for recurrent seizures after the first surgery are that invasive monitoring may not have covered the full extent of the epileptogenic zone and thus an incomplete resection of the epileptogenic zone was performed, limited resection was performed to preserve eloquent cortex, resection was not performed as initially planned, or a patient may have developed a secondary epileptogenic zone following resection. . $^{1,3,5,9,19,20,31}$

Multiple adult case series have reviewed resective reoperations after failed epilepsy surgery, finding that $20 \%-41 \%$ of patients attain seizure freedom..$^{6,723,24,27} \mathrm{Few}$ studies have addressed this issue in the pediatric population. Ramantani et al. ${ }^{21}$ and Shaver et al. ${ }^{26}$ reported on their outcomes for reoperation in children but did not include reevaluation prior to the second surgery. Bower et al. ${ }^{2}$ reported that their reevaluation prior to reoperation included MRI, subtraction ictal SPECT coregistered to MRI (SIS-
TABLE 4. Patients who were seizure free versus those with persistent seizures 1 year after the second surgery

\begin{tabular}{|c|c|c|c|}
\hline Parameter & $\begin{array}{l}\text { Seizure } \\
\text { Free }\end{array}$ & $\begin{array}{l}\text { Persistent } \\
\text { Seizures }\end{array}$ & $\begin{array}{c}p \\
\text { Value* }^{*}\end{array}$ \\
\hline No. of patients & 14 & 10 & \\
\hline Female sex & $7(50 \%)$ & $3(30 \%)$ & 0.327 \\
\hline Age at seizure onset in yrs & $3.38 \pm 3.22$ & $5.35 \pm 4.27$ & 0.210 \\
\hline Age at 1st surgery in yrs & $6.95 \pm 4.36$ & $8.67 \pm 4.67$ & 0.323 \\
\hline Age at 2 nd surgery in yrs & $10.46 \pm 4.76$ & $10.96 \pm 2.90$ & 0.771 \\
\hline Site of seizure focus & & & 0.186 \\
\hline Temporal & $5(36 \%)$ & $7(70 \%)$ & \\
\hline Extratemporal & $8(57 \%)$ & $2(20 \%)$ & \\
\hline Temporal \& extratemporal & $1(7 \%)$ & $1(10 \%)$ & \\
\hline Normal preop MRI & $2(14 \%)$ & $0(0 \%)$ & 0.493 \\
\hline Histology & & & 0.255 \\
\hline FCD & $4(29 \%)$ & $3(30 \%)$ & \\
\hline Tumor & $6(43 \%)$ & $1(10 \%)$ & \\
\hline Rasmussen encephalitis & $1(7 \%)$ & $1(10 \%)$ & \\
\hline Other & $1(7 \%)$ & $4(40 \%)$ & \\
\hline No discernable pathology & $2(14 \%)$ & $1(10 \%)$ & \\
\hline $\begin{array}{l}\text { Similar seizure semiology after } \\
\text { 1st surgery }\end{array}$ & $9(64 \%)$ & $6(60 \%)$ & 0.582 \\
\hline $\begin{array}{l}\text { Clustered dipoles in MEG } \\
\text { before } 2 \text { nd surgery } \dagger\end{array}$ & $10(71 \%)$ & $4(40 \%)$ & 0.080 \\
\hline $\begin{array}{l}\text { Invasive monitoring at } 2 \text { nd } \\
\text { surgery }\end{array}$ & $3(21 \%)$ & $4(40 \%)$ & 0.296 \\
\hline $\begin{array}{l}\text { Residual lesion identified after } \\
\text { 2nd surgery }\end{array}$ & $1(8 \%)$ & $1(10 \%)$ & 0.714 \\
\hline Type of 2nd surgery & & & 0.542 \\
\hline $\begin{array}{l}\text { Lesionectomy (\& recurrent } \\
\text { tumor resection) }\end{array}$ & $4(29 \%)$ & $1(10 \%)$ & \\
\hline Extended cortical resection & $5(36 \%)$ & $3(30 \%)$ & \\
\hline Lobectomy & $3(21 \%)$ & $5(50 \%)$ & \\
\hline Functional hemispherotomy & $2(14 \%)$ & $1(10 \%)$ & \\
\hline
\end{tabular}

Values expressed as mean \pm SD or as number (\%).

* Results of independent samples t-tests, chi-square analyses, or Fisher's exact test, as appropriate.

† Magnetoencephalography was completed for 21 children.

COM), and long-term VEEG in the majority of patients, with $20 \%$ of their patients also undergoing PET. All of our patients underwent MRI and VEEG and the majority underwent MEG. The MEG clustered dipoles have been shown to correlate with the ictal onset zone on intracranial EEG. ${ }^{18}$ At our institution, MEG is performed in approximately $85 \%$ of patients before the first surgery and has been shown to be concordant with the resection site in $77 \%$ of patients. ${ }^{22}$ Despite having MEG clustered dipoles prior to the first surgery, patients may have persistent seizures due to incomplete resection of the lesion or epileptogenic zone or because some patients may develop a secondary epileptogenic zone following the first surgery. A previous study by our group ${ }^{16}$ in a smaller cohort showed that removing the MEG clustered dipoles adjacent to the margins of previous resections, in addition to removing residual and/or recurrent lesion, achieved seizure-free outcome in $8(62 \%)$ 
of 13 patients who had undergone reoperation. In the present study, 21 patients underwent MEG prior to reoperation, and $10(71 \%)$ of 14 patients with MEG clustered dipoles were seizure free. We found that patients with MEG clustered dipoles trend toward a seizure-free outcome (71\% vs $40 \%$ ) following reoperation. Additional investigations prior to the second surgery included invasive monitoring. None of the 7 patients (29\%) who underwent invasive monitoring prior to the first surgery had invasive monitoring prior to reoperation. It is technically more challenging to place a subdural grid in patients who have had prior subdural grid monitoring. Seven (41\%) of the 17 patients who did not have invasive monitoring prior to the first surgery did undergo invasive monitoring prior to reoperation; 3 of these patients $(43 \%)$ were seizure free and $2(29 \%)$ had improvement in seizures following reoperation.

Some limitations of the present study should be noted. First, its retrospective nature limits our evaluation of the possible predictors of favorable seizure outcomes. Second, the patients included in this study underwent their first surgery between 2001 and 2013; technological improvements in MRI may help to improve detection of subtle lesions such as FCD and better define the extent of FCD, thereby improving outcome following first surgery and reoperations. Furthermore, newer surgical techniques such as MRI-guided laser therapy have been increasingly used for lesions such as hypothalamic hamartoma and may replace resective surgery for hypothalamic hamartoma. ${ }^{4,15}$ Because of the study's small sample size, we were unable to determine if outcomes have changed with time.

\section{Conclusions}

Our findings demonstrate that reoperation in children with recurrent seizures after the first epilepsy surgery could result in favorable seizure outcomes. We found that $14(58 \%)$ of 24 patients achieved a seizure-free outcome and that $18(75 \%)$ of 24 patients had improvement in seizures 1 year after reoperation. Patients who have persistent seizures at 1 year or less after the first epilepsy surgery should be evaluated for consideration of reoperation. In particular, those with residual lesion after the first surgery should undergo reevaluation and complete resection of the lesion to improve seizure outcome earlier. We also showed that there was a trend for a seizure-free outcome in patients with MEG dipole clusters. Data in this study suggest that reevaluation prior to reoperation should include MRI, VEEG, and MEG and perhaps invasive monitoring in selected patients, particularly those with no residual lesion and MEG that shows no dipole cluster.

\section{References}

1. Basheer SN, Connolly MB, Lautzenhiser A, Sherman EM, Hendson G, Steinbok P: Hemispheric surgery in children with refractory epilepsy: seizure outcome, complications, and adaptive function. Epilepsia 48:133-140, 2007

2. Bower RS, Wirrell EC, Eckel LJ, Wong-Kisiel LC, Nickels $\mathrm{KC}$, Wetjen NM: Repeat resective surgery in complex pediatric refractory epilepsy: lessons learned. J Neurosurg Pediatr 16:94-100, 2015

3. Cossu M, Lo Russo G, Francione S, Mai R, Nobili L, Sartori
I, et al: Epilepsy surgery in children: results and predictors of outcome on seizures. Epilepsia 49:65-72, 2008

4. Curry DJ, Gowda A, McNichols RJ, Wilfong AA: MR-guided stereotactic laser ablation of epileptogenic foci in children. Epilepsy Behav 24:408-414, 2012

5. Englot DJ, Han SJ, Rolston JD, Ivan ME, Kuperman RA, Chang EF, et al: Epilepsy surgery failure in children: a quantitative and qualitative analysis. J Neurosurg Pediatr 14:386-395, 2014

6. González-Martínez JA, Srikijvilaikul T, Nair D, Bingaman WE: Long-term seizure outcome in reoperation after failure of epilepsy surgery. Neurosurgery 60:873-880, 2007

7. Goodman RR: AES 2009 Annual Course: Reoperation for medically refractory epilepsy. Epilepsy Behav 20:241-246, 2011

8. Harris PA, Taylor R, Thielke R, Payne J, Gonzalez N, Conde JG: Research electronic data capture (REDCap) - a metadata-driven methodology and workflow process for providing translational research informatics support. J Biomed Inform 42:377-381, 2009

9. Hemb M, Velasco TR, Parnes MS, Wu JY, Lerner JT, Matsumoto JH, et al: Improved outcomes in pediatric epilepsy surgery: the UCLA experience, 1986-2008. Neurology 74:1768-1775, 2010

10. Holmes MD, Wilensky AJ, Ojemann LM, Ojemann GA: Predicting outcome following reoperation for medically intractable epilepsy. Seizure 8:103-106, 1999

11. Holowka SA, Otsubo H, Iida K, Pang E, Sharma R, Hunjan A, et al: Three-dimensionally reconstructed magnetic source imaging and neuronavigation in pediatric epilepsy: technical note. Neurosurgery 55:1226, 2004

12. Iida K, Otsubo H, Matsumoto Y, Ochi A, Oishi M, Holowka $\mathrm{S}$, et al: Characterizing magnetic spike sources by using magnetoencephalography-guided neuronavigation in epilepsy surgery in pediatric patients. J Neurosurg 102 (2 Suppl):187-196, 2005

13. Iida K, Otsubo H, Mohamed IS, Okuda C, Ochi A, Weiss SK, et al: Characterizing magnetoencephalographic spike sources in children with tuberous sclerosis complex. Epilepsia 46:1510-1517, 2005

14. Kiehna EN, Widjaja E, Holowka S, Carter Snead O III, Drake J, Weiss SK, et al: Utility of diffusion tensor imaging studies linked to neuronavigation and other modalities in repeat hemispherotomy for intractable epilepsy. J Neurosurg Pediatr 17:483-490, 2016

15. Lewis EC, Weil AG, Duchowny M, Bhatia S, Ragheb J, Miller I: MR-guided laser interstitial thermal therapy for pediatric drug-resistant lesional epilepsy. Epilepsia 56:1590-1598, 2015

16. Mohamed IS, Otsubo H, Ochi A, Elliott I, Donner E, Chuang $\mathrm{S}$, et al: Utility of magnetoencephalography in the evaluation of recurrent seizures after epilepsy surgery. Epilepsia 48:2150-2159, 2007

17. Pati S, Abla AA, Rekate HL, Ng YT: Repeat surgery for hypothalamic hamartoma in refractory epilepsy. Neurosurg Focus 30(2):E3, 2011

18. RamachandranNair R, Otsubo H, Shroff MM, Ochi A, Weiss SK, Rutka JT, et al: MEG predicts outcome following surgery for intractable epilepsy in children with normal or nonfocal MRI findings. Epilepsia 48:149-157, 2007

19. Ramantani G, Kadish NE, Brandt A, Strobl K, Stathi A, Wiegand G, et al: Seizure control and developmental trajectories after hemispherotomy for refractory epilepsy in childhood and adolescence. Epilepsia 54:1046-1055, 2013

20. Ramantani G, Kadish NE, Strobl K, Brandt A, Stathi A, Mayer H, et al: Seizure and cognitive outcomes of epilepsy surgery in infancy and early childhood. Eur J Paediatr Neurol 17:498-506, 2013

21. Ramantani G, Strobl K, Stathi A, Brandt A, Schubert-Bast 
S, Wiegand G, et al: Reoperation for refractory epilepsy in childhood: a second chance for selected patients. Neurosurgery 73:695-704, 2013

22. Rubinger L, Chan C, D'Arco F, Moineddin R, Muthaffar O, Rutka JT, et al: Change in presurgical diagnostic imaging evaluation affects subsequent pediatric epilepsy surgery outcome. Epilepsia 57:32-40, 2016

23. Salanova V, Markand O, Worth R: Temporal lobe epilepsy: analysis of failures and the role of reoperation. Acta Neurol Scand 111:126-133, 2005

24. Schulz R, Hoppe M, Boesebeck F, Gyimesi C, Pannek HW, Woermann FG, et al: Analysis of reoperation in mesial temporal lobe epilepsy with hippocampal sclerosis. Neurosurgery 68:89-97, 2011

25. Schwartz TH, Spencer DD: Strategies for reoperation after comprehensive epilepsy surgery. J Neurosurg 95:615-623, 2001

26. Shaver EG, Harvey AS, Morrison G, Prats A, Jayakar P, Dean P, et al: Results and complications after reoperation for failed epilepsy surgery in children. Pediatr Neurosurg 27:194-202, 1997

27. Siegel AM, Cascino GD, Meyer FB, McClelland RL, So EL, Marsh WR, et al: Resective reoperation for failed epilepsy surgery: seizure outcome in 64 patients. Neurology 63:22982302, 2004

28. Spencer S, Huh L: Outcomes of epilepsy surgery in adults and children. Lancet Neurol 7:525-537, 2008

29. Steinbok P, Gan PY, Connolly MB, Carmant L, Barry Sinclair D, Rutka J, et al: Epilepsy surgery in the first 3 years of life: a Canadian survey. Epilepsia 50:1442-1449, 2009

30. Téllez-Zenteno JF, Dhar R, Wiebe S: Long-term seizure outcomes following epilepsy surgery: a systematic review and meta-analysis. Brain 128:1188-1198, 2005

31. Terra-Bustamante VC, Inuzuca LM, Fernandes RM, Funayama S, Escorsi-Rosset S, Wichert-Ana L, et al: Temporal lobe epilepsy surgery in children and adolescents: clinical characteristics and post-surgical outcome. Seizure 14:274-281, 2005

32. Wieser HG, Blume WT, Fish D, Goldensohn E, Hufnagel A, King D, et al: ILAE Commission Report. Proposal for a new classification of outcome with respect to epileptic seizures following epilepsy surgery. Epilepsia 42:282-286, 2001

33. Winkler PA, Kudernatsch M, Pieper T, Keßler-Uberti S, Staudt M, Holthausen H: Reoperations and second stepprocedures in pediatric epilepsy surgery-indications, new techniques, limitations and results. Neuropediatrics 42:V10, 2011

34. Wyler AR, Hermann BP, Richey ET: Results of reoperation for failed epilepsy surgery. J Neurosurg 71:815-819, 1989

\section{Disclosures}

The authors report no conflict of interest concerning the materials or methods used in this study or the findings specified in this paper.

\section{Author Contributions}

Conception and design: Widjaja, Muthaffar, Go, Snead, Rutka. Acquisition of data: Rubinger. Analysis and interpretation of data: Widjaja, Muthaffar, Puka. Drafting the article: Muthaffar, Puka. Critically revising the article: all authors. Reviewed submitted version of manuscript: all authors. Approved the final version of the manuscript on behalf of all authors: Widjaja. Statistical analysis: Puka. Study supervision: Widjaja.

\section{Correspondence}

Elysa Widjaja, Hospital for Sick Children, Diagnostic Imaging, 555 University Ave., Toronto, ON M5G 1X8, Canada. email: elysa.widjaja@sickkids.ca. 